\title{
A TEXTILE ANTENNA BASED ON HIGH-PERFORMANCE FABRICS
}

\author{
C. Hertleer*, H. Rogier $^{\dagger}$, L. Vallozzi ${ }^{\dagger}$ and F. Declercq $^{\dagger}$ \\ * Ghent University, Department of Textiles, \\ Technologiepark 907, 9052 Zwijnaarde, Belgium, \\ Tel: +32926495 30, Carla.Hertleer@UGent.be \\ ${ }^{\dagger}$ Ghent University, Department of Information Technology, \\ Sint-Pietersnieuwstraat 41, 9000 Gent, Belgium
}

Keywords: wearable electronics, textile antenna, intelligent textile.

\begin{abstract}
This paper reports a multilayer textile microstrip patch antenna for integration into protective clothing for professional workers since it is made out of a high performance aramid fabric. It is designed to operate in the $2.45 \mathrm{GHz}$ Industrial, Scientific and Medical (ISM) band for short range communication to transmit vital signs, activity and environmental conditions to a nearby base station. Electrotextiles are used for the antenna patch and ground plane. Integration of the textile antenna into the protective clothing requires a finite ground plane and reliable operation in the vicinity of the body. Furthermore the patch may bend. A rectangular ring topology is proposed to preserve the antenna characteristics in real-life applications. This research paves the way for a new generation of protective clothing.
\end{abstract}

\section{Introduction}

The main purpose of clothing is protecting the human body against environmental conditions. Professional workers like firemen benefit to a large extent from the scientific development such as the introduction of high performance fibers that have been achieved in the previous century. The 911 events in the US raised the awareness of the importance of equipping fire fighters, paramedics and other rescue workers with the most advanced materials. Furthermore, the development of wearable intelligent textile systems has altered the concept of clothing. New generation garments are not only used as carrier but also as basic material for the required components such as sensors and thus capable of monitoring the wearer's vital signs, activity and environmental parameters $[3,4]$. These intelligent textiles have uncovered the need for wireless communication systems that are unnoticeably embedded into clothing $[1,6]$.

In that perspective, a textile antenna based on speciality fabrics is required and will be presented in this paper. Implementing the antenna into clothing will maintain wearing comfort while supporting the operability. However, additional requirements have to be taken into account for the antenna design. The material selection is a crucial step when designing an antenna for integration into protective clothing. The textile antennas proposed in [6,7] are made out of a lowcost fleece fabric which is not suitable for integration into a fire fighter garment because it is not flame resistant unlike the aramid woven fabric. Antennas mounted onto fleece fabric provide a better antenna efficiency and gain in comparison to the aramid fabric because of the lower permittivity, smaller loss tangent and larger thickness of the fleece. This also results in a larger impedance bandwidth of the fleece-based antenna. However, the use of a high-performance aramid fabric is inevitable for integration of the textile antenna into protective clothing of professional workers. Therefore, the antenna was mounted on a multilayered aramid fabric to provide sufficient impedance bandwidth and gain without harming the flexibility of the fabric too much. This multilayered aramid substrate is also dimensionally more stable than the compressible fleece fabric, which allows applying the antenna in outer garments meant for harsh environments. This research was carried out in the framework of the European Integrated Project Proetex [5] that promotes the development of wearable textile systems to support the work of the emergency worker and firemen.

Our paper is organized as follows: In the next section the rectangular ring topology of the patch for maintaining the antenna characteristics in real-life applications is motivated. Moreover, the placement of the antenna in the protective clothing is outlined. Section 3 describes the antenna's reflection coefficient measurements using an Agilent 8714 vector network analyzer, transmission measurements in the anechoic chamber and the on-body gain measurements to prove the robustness of the antenna characteristics in real-life applications.

\section{Antenna geometry and design}

Our point of departure was a microstrip patch antenna built up as a multilayer textile structure and applied for off-body short range communication. We focus on Wireless Local Area Networks (WLAN) that operate in the $2.45 \mathrm{GHz}$ ISM band and are based on protocols such as Zigbee, Bluetooth, WiFi 
and Wireless USB. An antenna with a $-10 \mathrm{~dB}$ return loss over the entire ISM band (2.4-2.4835 GHz) had to be obtained. To improve reception in real-life applications, where the wearer is mobile and continuously changing his orientation with respect to the receiving antenna, linear polarization had to be avoided. To achieve these specifications, a single feed rectangular ring topology $[7,8]$ was chosen. Placing the feed point on a diagonal of the patch avoids linear polarization by means of excitation of the two orthogonal modes TM10 and TM01. The bandwidth of the design was increased by choosing slightly different values for the patch length $L$ and width $W$, causing the modes TM10 and TM01 to resonate at different frequencies. The bandwidth was further enhanced by inserting a slot with dimensions $l$ and $w$. Since the antenna is designed for integration into a fire fighter's jacket, the outer shell fabric of such a garment was chosen as antenna substrate. This is a high performance aramid woven fabric with a thickness of $0.40 \mathrm{~mm}$. This fabric is part of the flameresistant outer shell. However, because we require a bandwidth of over $83.5 \mathrm{MHz}$, an antenna substrate with a sufficient thickness is needed. Therefore, we assembled four aramid layers using an adhesive sheet, resulting in an overall thickness of $1.67 \mathrm{~mm}$. The substrate properties are mentioned in Table 1. The geometry of the patch and the location of the feed point were optimized using the commercial field simulator Momentum ${ }^{\circledR}$ from Agilent Technologies. The resulting topology of the rectangular-ring textile antenna is depicted in Figure 1 and the dimensions are given in Table 1.

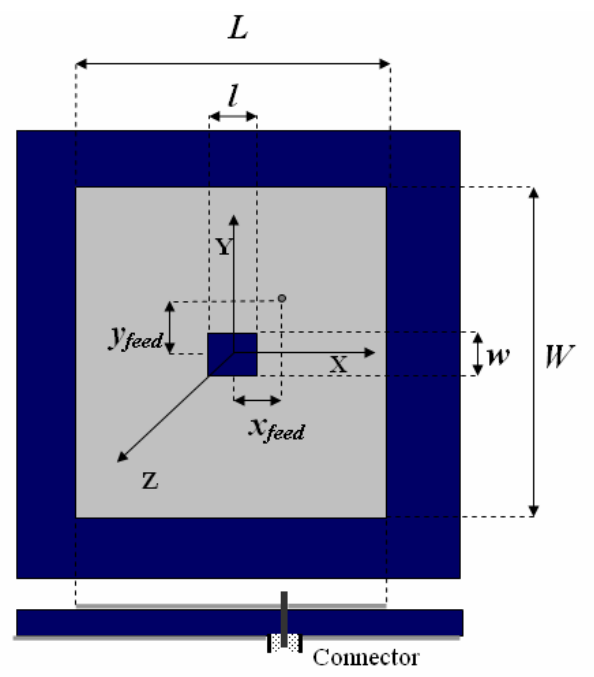

Figure 1: Geometry of the rectangular ring textile antenna.

The antenna is equipped with a ground plane shielding the body from the radiation. However, this ground plane should be kept as small as possible to maintain the wearing comfort of the user. Here a ground plane of 10 to $10 \mathrm{~cm}$ is provided. The patch and ground plane are built out of a commercially available electrotextile with a very high conductivity (surface resitivity $<0.1 \Omega / \mathrm{sq}$ ). The antenna was excited by means of a $50 \Omega$ SMA feed, which was carefully soldered onto the antenna. The simple rectangular ring topology also allows easy cutting out of the textile material.
The proposed antenna is designed for integration into the outer fire fighter garment. Since this is a multilayer assembly, two types of positioning have to be determined: between what layers and where on the garment. The antenna will be wired to the monitoring system within the garment and the monitoring sensors are embedded in the underwear of the rescue workers [2]. Therefore it was decided to locate it underneath the moisture barrier and the thermal barrier layer. This avoids routing wires through the combined moisture and thermal barrier layer that is responsible for the waterproof property of the garment. Underneath the antenna's ground plane is the inner lining of the garment as shown in Figure 2. The antenna placement on the garment was preferred in the area of the shoulders or the upper arm as depicted in Figure 3, because of the minimal risk of creasing and wrinkling in these areas.

\begin{tabular}{|c|c|c|c|c|c|}
\hline \multicolumn{4}{|c|}{ Patch (mm) } & \multirow{2}{*}{\multicolumn{2}{|c|}{$\begin{array}{c}\text { Aramid } \\
\text { substrate } \\
\text { (4 layers) }\end{array}$}} \\
\hline$L$ & 43 & $W$ & 41 & & \\
\hline$l$ & 8 & $w$ & 7 & $\mathrm{~h}(\mathrm{~mm})$ & 1.67 \\
\hline \multicolumn{2}{|c|}{$\mathrm{X}_{\mathrm{feed}}$} & \multicolumn{2}{|c|}{7} & $\varepsilon_{\mathrm{r}}$ & 1.85 \\
\hline \multicolumn{2}{|c|}{$y_{\text {feed }}$} & \multicolumn{2}{|c|}{6} & $\tan \delta$ & 0.015 \\
\hline
\end{tabular}

Table 1: Antenna dimensions and substrate properties.

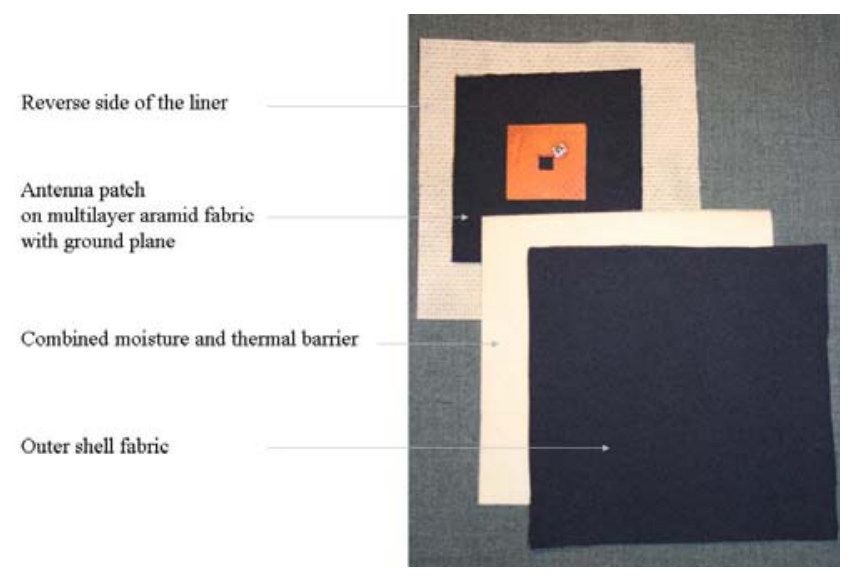

Figure 2: Positioning of the antenna with regard to the assembly of the garment.

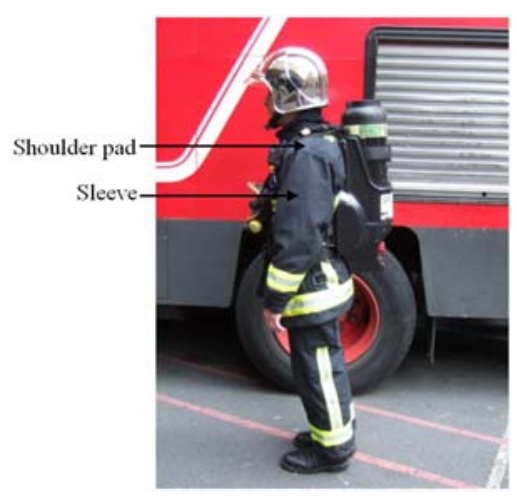

Figure 3: Possible location of the antenna on the garment. 


\section{Simulation and measurement results}

For the reflection measurement an Agilent 8714 ET Network Analyzer was used. First the return loss of the antenna was measured in planar state, in order to compare the simulation results with the measurement. Second, the return loss of the bent antenna was measured to evaluate its bandwidth robustness. Therefore, the antenna was curved over a circular cylinder with a diameter of $8 \mathrm{~cm}$, which corresponds with an arm or a shoulder. Simulation and measurement of the rectangular ring textile antenna are compared in Figure 4 and show an excellent agreement. A robust design is obtained by increasing the bandwidth up to $100 \mathrm{MHz}$. That is because we expect a shift in resonance frequencies in real-life applications due to bending and geometrical design tolerances of the patch. Both in planar and bent state, the measured bandwidth of the textile antenna are found to be slightly over the $100 \mathrm{MHz}$.

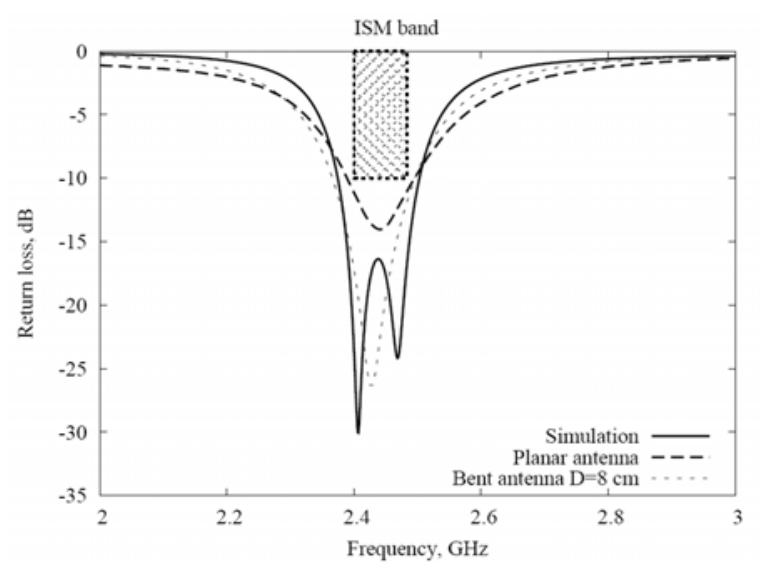

Figure 4: Measured and simulated return loss.

For the transmission measurements an HP8510C Network Analyzer was applied. The set up in the anechoic room consisted of a standard gain horn antenna as transmitting antenna and the textile antenna, mounted on an Orbit/Fr positioning system, as receiving antenna as illustrated in Figure 5. In order to evaluate the presence of the additional layers covering the antenna in real-life circumstances, the transmission parameters were consecutively measured without and with the extra layers.

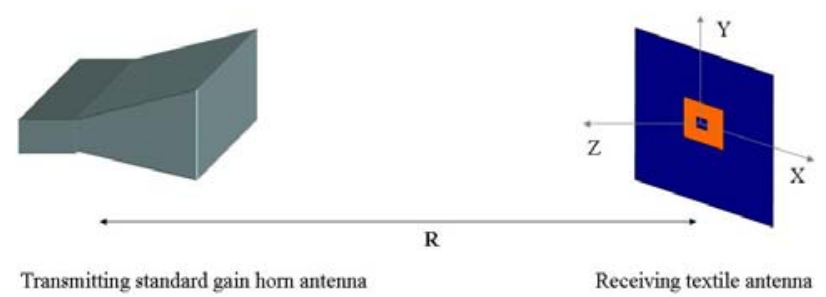

Figure 5: Measurement setup transmission measurements

This resulted in four types of measurements: uncovered and covered antenna both when the antenna was in planar and bent state. For each line-up, transmission measurements were executed both in the $\mathrm{XZ}$-and the $\mathrm{YZ}$ plane. The antenna gain resulting from these data is presented in Figure 6 and Figure 7 for the flat and bent antenna respectively. The gain pattern shows us how the radiated power is redistributed in all directions and most of the power is radiated in the direction perpendicular to the antenna (z-direction). A comparison between the gain pattern in Figure 6 and Figure 7 shows us that in the case of bending the antenna, the radiated power is distributed more evenly in the hemisphere ranging from $-90^{\circ}$ to $90^{\circ}$. This, however, will not diminish the quality of the communication link. Table 2 depicts the maximum gain and the $-3 \mathrm{~dB}$ beam width for the four different measurement configurations.

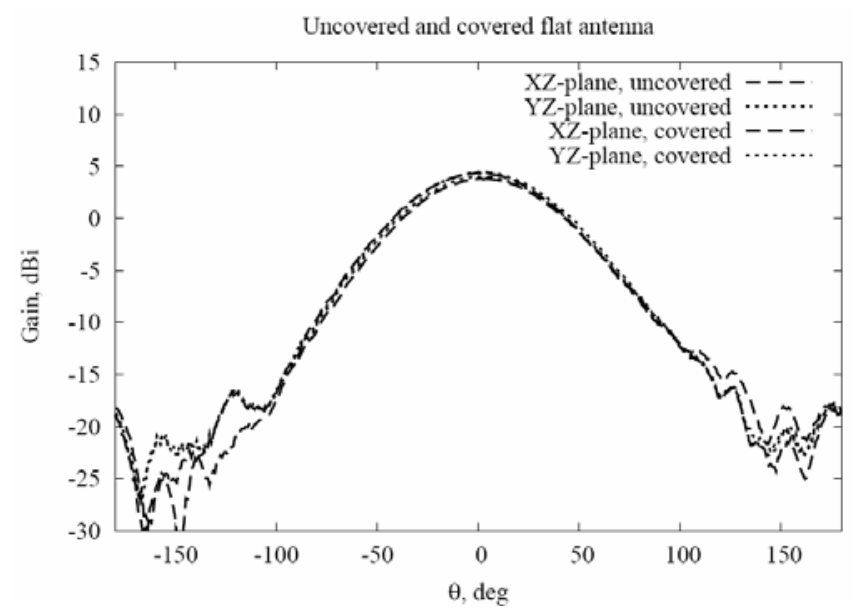

Figure 6: Measured antenna gain of the uncovered and covered flat antenna at $2.45 \mathrm{GHz}$.

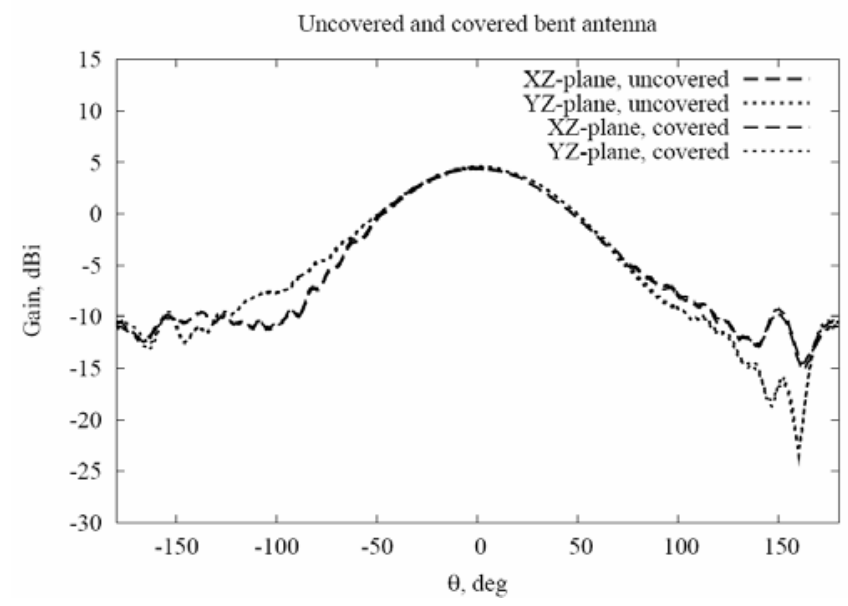

Figure 7: Measured antenna gain of the uncovered and covered bent antenna at $2.45 \mathrm{GHZ}$

Figure 8 compares the simulation results of the antenna, in which only substrate losses were included, with the measured antenna gain of the uncovered flat planar antenna and reveals that there is a good agreement. The difference between the simulated gain and measurement gain is due to the fact that conductor losses were not taken into account during 
simulations. No backward radiation is present in the simulation results because of the infinite ground plane. The simulated gain from the lossless case results in a maximum directivity of $7.7 \mathrm{dBi}$ in the $\mathrm{z}$-direction, yielding an antenna efficiency of $47 \%$. This low but acceptable efficiency is due to the substrate and conductor losses, which are hard to avoid.

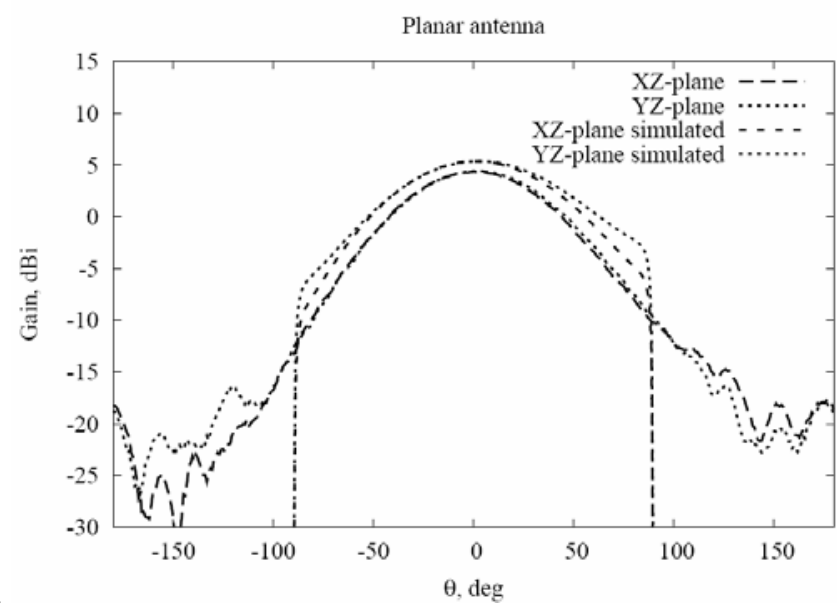

Figure 8: Simulated versus measured gain of the uncovered planar antenna at $2.45 \mathrm{GHz}$.

\begin{tabular}{|c|c|c|c|c|}
\hline \multirow{2}{*}{} & \multicolumn{2}{|c|}{$\begin{array}{c}\text { Maximum gain } \\
\text { (dB) }\end{array}$} & \multicolumn{2}{c|}{$\begin{array}{c}\text {-3dB beam } \\
\text { width (deg) }\end{array}$} \\
\cline { 2 - 5 } & $\begin{array}{c}\text { XZ- } \\
\text { plane }\end{array}$ & $\begin{array}{c}\text { YZ- } \\
\text { plane }\end{array}$ & $\begin{array}{c}\text { XZ- } \\
\text { plane }\end{array}$ & $\begin{array}{c}\text { YZ- } \\
\text { plane }\end{array}$ \\
\hline $\begin{array}{c}\text { Planar - } \\
\text { uncovered }\end{array}$ & 4.36 & 4.45 & 71.1 & 70.2 \\
\hline $\begin{array}{c}\text { Planar - } \\
\text { covered }\end{array}$ & 3.83 & 4.04 & 70.2 & 70.2 \\
\hline $\begin{array}{c}\text { Bent - } \\
\text { uncovered }\end{array}$ & 4.49 & 4.61 & 76.5 & 78.3 \\
\hline $\begin{array}{c}\text { Bent - } \\
\text { covered }\end{array}$ & 4.37 & 4.50 & 76.5 & 79.2 \\
\hline
\end{tabular}

Table 2: Maximum gain and $3 \mathrm{~dB}$ beam width of the antenna.

From the results described above we conclude that neither covering nor bending the antenna has a significant influence on its gain, since the differences in antenna gain are of the order of the measurement error. There is, however, a considerable increase of $-3 \mathrm{~dB}$ main beam width, which means that the antenna becomes less directional and implies that the antenna is capable of detecting a broader range of incoming rays. Finally, on-body measurements were performed by attaching the covered antenna on a real-life application position such as the shoulder of the user as depicted in Figure 9. The resulting gain of the on body measurement is given in Figure 10, showing us that the antenna gain is decreased in comparison with the off-body measurements depicted in Table 2. However, the gain remains above the $3 \mathrm{~dB}$ in the entire ISM band.

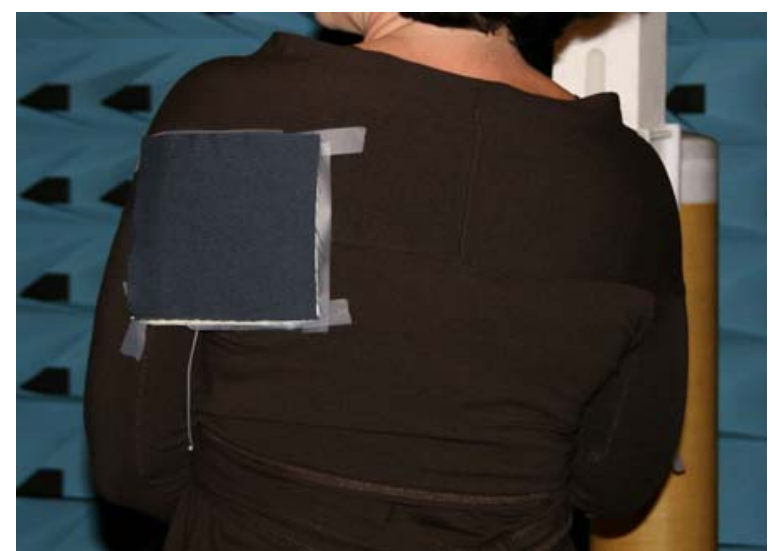

Figure 9: Measurement setup on-body gain measurement.

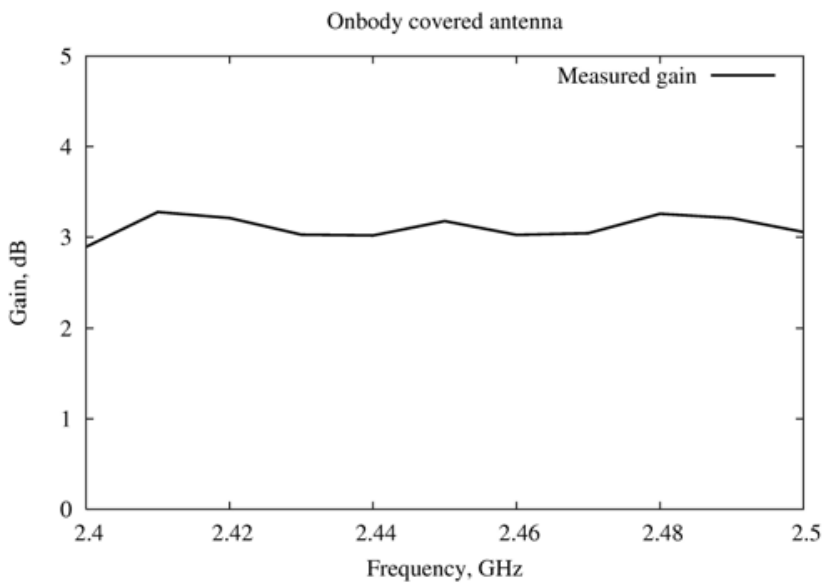

Figure 10: On body gain measurement.

\section{Conclusion}

The proposed rectangular-ring antenna based on a flameresistant aramid fabric provides sufficient gain to operate from an inner layer of a fire fighters' outer garment. The antenna was designed to operate in the $2.45 \mathrm{GHz}$ ISM band for Local Area Networks. Assembling four layers of the aramid fabric provides an antenna substrate with sufficient thickness rigidity without harming the wear comfort. Even in bent state the transmission and reflection measurement show us that the antenna guarantees a reliable wireless communication link between the wearer and the base station.

\section{Acknowledgements}

This research was accomplished within the framework of the ProeTex project and the authors would like to acknowledge the European Commission for funding it (FP6-2004-IST-4026987). H. Rogier is a postdoctoral researcher of the FWO$\mathrm{V}$.

\section{References}

[1] M. Klemm, I. Locher, G. Tröster, "A Novel Circularly Polarized Textile Antenna for Wearable Applications", 
Proc. of $7^{\text {th }}$ European Microwave Week, pp. 137-140, (2004)

[2] M. Pacelli, G. Loriga, "Flat Knitted Sensors for Respiration Monitoring", IEEE International Symposium on Industrial Electronics, pp. 2838-2841, (2007)

[3] R. Paradiso, G. Loriga, N. Taccini, "A Wearable Health Care System Based on Knitted Integrated Sensors", IEEE Transactions on Information Technology in Biomedicine, 9, $\mathrm{n}^{\circ} 3$ pp. 337-344, (2005)

[4] S. Park, S. Jayaraman, "Enhancing the quality of life through wearable technology", IEEE Eng. Med. Biol. Mag., 22, n 3, pp. 41-48, (2003)

[5] www.proetex.org

[6] P. Salonen, H. Hurme, "A novel fabric WLAN antenna for wearable applications", IEEE Antennas and Propagation Society International Symposium, 2, pp. 700-703, (2003)

[7] A. Tronquo, H. Rogier, C. Hertleer, and L. Van Langenhove. "A Robust Planar Textile Antenna for Wireless Body LANs Operating in the $2.45 \mathrm{GHz}$ ISM band“", Electronics Letters, 42, pp. 142-143, (2006)

[8] G. Vermeeren, H. Rogier, F. Olyslager, D. De Zutter. "Simple low-cost planar antenna for indoor communication under the Bluetooth protocol", Electronics Letters, 37, pp. 1153-1154, (2001) 

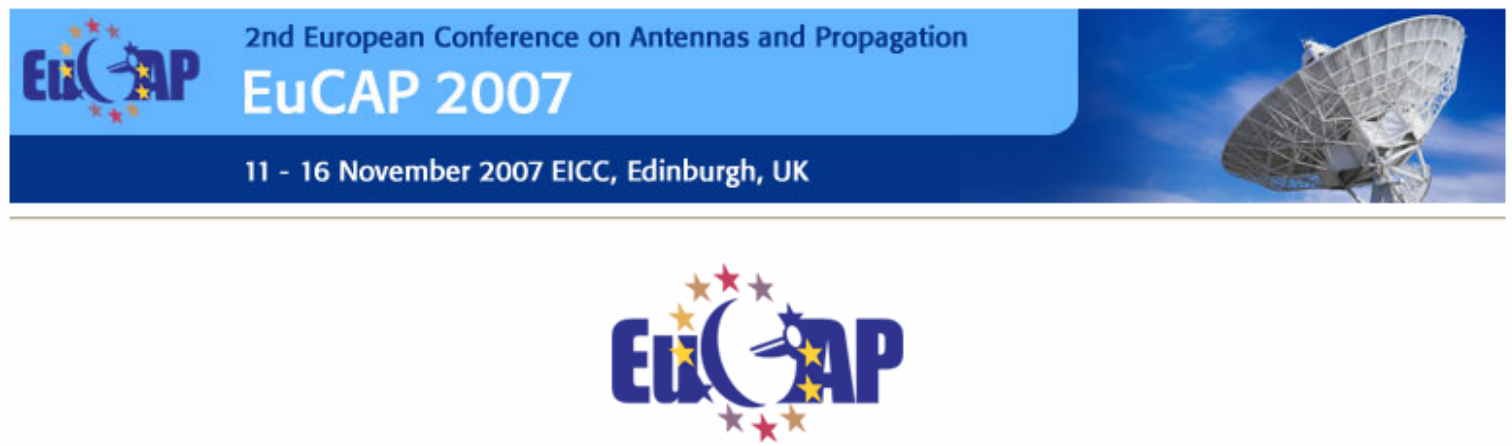

Welcome to

\section{The Second European Conference on Antennas and Propagation EuCAP 2007}

11 - 16 November 2007, The EICC, Edinburgh, UK

Organised by the Institution of Engineering and Technology on behalf of EurAAP AISBL

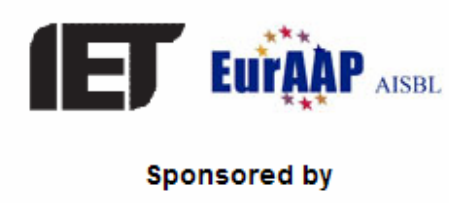

SEMCAD - Cesa

ISBN 978-0-86341-842-6 